\title{
Precision nitrogen management in drip irrigated maize (Zea mays L.)
}

\author{
Yogananda Shivalli Boregowda ${ }^{1^{*}}$, Thimmegowda Puttavenkategowda ${ }^{2}$, Prakash Salekoplu Sannegowda ${ }^{3}$ and Shruthi \\ Goravale Kempegowda ${ }^{1}$ \\ ${ }^{1}$ Department of Agronomy, College of Agriculture, V. C. Farm, Mandya-571 405, India \\ ${ }^{2}$ Senior Farm Superintendent, Zonal Agricultural Research Station, V. C. Farm, Mandya - 571 405, India \\ ${ }^{3}$ Dept. of Soil Science \& Agricultural Chemistry, College of Agriculture, V. C. Farm, Mandya-571 405, India
}

\begin{abstract}
The field experiment was conducted during kharif 2014 and 2015 at ZARS, V. C. Farm, Mandya to study the effect of precision nitrogen management on growth, yield and economics of drip irrigated maize. There were 9 treatments replicated thrice in Randomized Complete Block Design. Among the various treatments, drip fertigation of nitrogen through Soil Plant Analysis Development (SPAD) meter sufficiency index of 95-100 per cent under paired row (30/90) recorded significantly higher growth and yield parameters viz., plant height, LAl, total dry matter production, cob length, number of rows per cob, number of kernels per cob and cob weight along with kernel (85.73 and $86.78 \mathrm{q} / \mathrm{ha})$ and stover yield (140.4 and $145.3 \mathrm{q} / \mathrm{ha})$ during both the years of experimentation as compared to UAS Bangalore package with surface irrigation and normal spacing of $60 \mathrm{~cm} \times 30 \mathrm{~cm}$ and was being on par with nitrogen management through Leaf Colour Chart (LCC) 6, SPAD sufficiency index of 90-95 per cent and LCC 5 . The SPAD sufficiency index of 95-100 per cent registered higher net returns (Rs. 69,634 and Rs. 77,614 , respectively) and B:C ratio (2.82 and 3.00, respectively) followed by LCC 6 .
\end{abstract}

\section{INTRODUCTION}

Maize (Zea mays L.) has becoming very popular cereal crop in India because of the increasing market price and high production potential in both under irrigated as well as rainfed conditions. It occupies an important role next to rice and wheat in the farming sector and macroeconomy of the agrarian countries. It is third most important cereal crop in India after rice and wheat that occupied about 9.86 million hectares producing 26.26 million tons with an average productivity of $2664 \mathrm{~kg} \mathrm{ha}^{-1}$ during 2017-18 [1]. In India, twenty six per cent of the total maize produced being consumed as food by human beings, twelve per cent for starch extraction, two per cent seed and remaining sixty per cent is being used for animal and poultry feed industry [13]. The productivity of maize in a region is determined by several factors including nitrogen as one of the important factor. Application of higher level of $\mathrm{N}$-fertilizer is very common among Indian farmers, which attribute to positive growth response to $\mathrm{N}$ application [2]. Furthermore, large field to- field variability of soil $\mathrm{N}$ supply restricts efficient use of $\mathrm{N}$ fertilizer when broadbased blanket fertilizer $\mathrm{N}$ recommendations are used. When $\mathrm{N}$ application is not synchronized with crop demand, $\mathrm{N}$ losses from the soil plant system are large leading to low $\mathrm{N}$ use efficiency [15]. There is a need to synchronize $\mathrm{N}$ fertilizer application with plant need to optimize the nutrient use and minimize environmental

\footnotetext{
*Corresponding author: sbyogananda@gmail.com
}

pollution. Successful results in assessing $\mathrm{N}$ need at midseason are found in several studies [10]. Effective management of fertilizer, particularly $\mathrm{N}$ is a major challenge for researchers and as well as for producers especially under drip irrigation condition. Hence, there is need for precision nitrogen management in maize by using tools like LCC (Leaf Colour Chart) and SPAD (Soil Plant Analysis Development) meter for better utilization of nitrogen and also to obtain optimum yield. Considering the benefits of these tools, a field experiment was laid out with an objective to study the effect of precision nitrogen management on growth, yield, nitrogen use efficiency and economics in drip irrigated maize.

\section{MATERIAL AND METHODS}

A study was conducted during Kharif 2014 and 2015 at Zonal Agricultural Research Station, V. C. Farm, Mandya $\left(11^{0} 30^{\prime}\right.$ to $13^{\circ} 05^{\prime} \mathrm{N}$ latitude and $76^{0} 05^{\prime}$ to $77^{\circ}$ $45^{\prime}$ East longitude with an altitude of 695 meters above mean sea level). The soil of the experimental site was red sandy loam in texture with a $\mathrm{pH}$ of $6.60,0.40$ per cent organic carbon, $230.8 \mathrm{~kg} \mathrm{ha}^{-1}$ of available soil nitrogen, $41.9 \mathrm{~kg} \mathrm{ha}^{-1}$ of phosphorus and $146.2 \mathrm{~kg} \mathrm{ha}^{-1}$ of potassium content. The experiment consisted of 9 treatments viz., $\mathrm{T}_{1}$ : Nitrogen management through LCC3, $\mathrm{T}_{2}$ : Nitrogen management through LCC4, $\mathrm{T}_{3}$ : Nitrogen management through LCC5, $\mathrm{T}_{4}$ : Nitrogen management 
through LCC6, $\mathrm{T}_{5}$ : Nitrogen management through SPAD sufficiency index 85- 90 per cent, $\mathrm{T}_{6}$ : Nitrogen management through SPAD sufficiency index 90- 95 per cent, $\mathrm{T}_{7}$ : Nitrogen management through SPAD sufficiency index 95- 100 per cent, $\mathrm{T}_{8}$ : RDF with surface irrigation and paired row planting $(30 / 90 \mathrm{~cm}), \mathrm{T}_{9}$ : UAS (B) package with surface irrigation and normal spacing $(60 \mathrm{~cm} \times 30 \mathrm{~cm}$ ) laid out in Randomized Complete Block Design and replicated thrice. For $T_{1}$ to $T_{7}$ treatments, basal dose of $10 \mathrm{~kg} \mathrm{~N} \mathrm{ha}^{-1}$ was applied and remaining $\mathrm{N}$ was top dressed by using LCC and SPAD sufficiency index from 14 DAS to 50 per cent tasseling. In addition, for all the treatments full dose of $\mathrm{P}$ and $\mathrm{K}$ was applied as basal. But for the T8 and T9 treatments $75 \mathrm{~kg} \mathrm{~N}$ ha-1was applied as basal dose at 30 DAS. SPAD sufficiency index was calculated using the below formula:

$$
\begin{gathered}
\text { SPAD } \\
\begin{array}{c}
\text { Sufficiency } \\
\text { Index }
\end{array}
\end{gathered}=\frac{\begin{array}{c}
\text { Average bulk reading } \\
\text { Average reference } \\
\text { strip reading }
\end{array}}{\text { X }} 100
$$

The periodic growth and yield observations were taken in 5 randomly tagged plants in net plot area. Nitrogen uptake by crop at harvest was determined by Kjeldahl's method as described by [7]. Economics is calculated by considering the prices in the market and also by depreciation over the drip irrigation system. Fischer's method of analysis of variance was used for analysis and interpretation of the data as outlined by [6]. The level of significance used in ' $F$ ' and ' $T$ ' tests was $\mathrm{p}=0.05$. Critical differences were calculated wherever ' $\mathrm{F}$ ' test was significant.

\section{RESULTS AND DISCUSSION}

The growth parameters viz., plant height and total dry matter production at harvest and LAI at 90 DAS varied significantly among the $\mathrm{N}$ management practices during both the years of experimentation. $\mathrm{N}$ management through SPAD sufficiency index 95-100 per cent recorded significantly higher plant height (203.8 and $210.3 \mathrm{~cm}$, respectively) as compared to UAS (B) package (178.1 and $182.6 \mathrm{~cm}$, respectively). However, it was at par with nitrogen management through LCC 6 (201.9 and $206.5 \mathrm{~cm}$, respectively), SPAD sufficiency index 90-95 per cent (199.1 and $205.6 \mathrm{~cm}$, respectively) and LCC 5 (198.1 and $195.8 \mathrm{~cm}$, respectively). Whereas, the lowest plant height was observed in LCC 3 (164.9 and $170.2 \mathrm{~cm}$, respectively). Similarly the total dry matter production at harvest was also significantly higher in $\mathrm{N}$ management through SPAD sufficiency index 95100 per cent (325.7 and $328.9 \mathrm{~g} /$ plant, respectively) and was being on par with $\mathrm{N}$ management through LCC 6 , SPAD sufficiency index 90-95 per cent and LCC 5. This increased plant height and total dry matter production in these treatments were mainly attributed to enhanced Leaf Area Index due to production of more number of leaves and leaf area resulted in increased synthesis of photosynthates. These results are in agreement with the findings of [8] in rice (Table 1).
Table 1. Growth and yield parameters of drip irrigated maize as influenced by precision nitrogen management practices

\begin{tabular}{|c|c|c|c|c|c|c|c|c|}
\hline \multirow[t]{2}{*}{$\begin{array}{c}\text { Trea } \\
t \\
\text { ment } \\
s\end{array}$} & \multicolumn{2}{|c|}{$\begin{array}{c}\text { Plant } \\
\text { height } \\
(\mathrm{cm})\end{array}$} & \multicolumn{2}{|c|}{$\begin{array}{c}\text { Leaf } \\
\text { Area } \\
\text { Index } \\
\text { at } 90 \\
\text { DAS } \\
\end{array}$} & \multicolumn{2}{|c|}{$\begin{array}{c}\text { Total } \\
\text { Dry } \\
\text { Weight } \\
\text { (g plant } \\
\text { 1 }^{-} \text {) }\end{array}$} & \multicolumn{2}{|c|}{$\begin{array}{c}\text { Cob } \\
\text { length } \\
(\mathrm{cm})\end{array}$} \\
\hline & $\begin{array}{l}20 \\
14 \\
\end{array}$ & $\begin{array}{c}201 \\
5\end{array}$ & $\begin{array}{l}20 \\
14 \\
\end{array}$ & $\begin{array}{l}20 \\
15 \\
\end{array}$ & $\begin{array}{c}201 \\
4 \\
\end{array}$ & $\begin{array}{c}201 \\
5 \\
\end{array}$ & $\begin{array}{l}20 \\
14 \\
\end{array}$ & $\begin{array}{c}201 \\
5 \\
\end{array}$ \\
\hline $\mathrm{T}_{1}$ & $\begin{array}{l}16 \\
4 . \\
9\end{array}$ & $\begin{array}{c}170 . \\
2\end{array}$ & $\begin{array}{c}5 . \\
77\end{array}$ & $\begin{array}{c}5 . \\
82\end{array}$ & $\begin{array}{c}212 \\
.2\end{array}$ & $\begin{array}{c}220 \\
.3\end{array}$ & $\begin{array}{c}10 \\
.1\end{array}$ & $\begin{array}{c}10 \\
2\end{array}$ \\
\hline $\mathrm{T}_{2}$ & $\begin{array}{l}17 \\
5 . \\
6\end{array}$ & $\begin{array}{c}178 . \\
4\end{array}$ & $\begin{array}{c}6 . \\
28\end{array}$ & $\begin{array}{c}6 . \\
32\end{array}$ & $\begin{array}{c}253 \\
.9\end{array}$ & $\begin{array}{c}256 \\
.9\end{array}$ & $\begin{array}{c}11 \\
.1\end{array}$ & $\begin{array}{c}11 . \\
6\end{array}$ \\
\hline $\mathrm{T}_{3}$ & $\begin{array}{c}19 \\
8 . \\
1\end{array}$ & $\begin{array}{c}195 . \\
8\end{array}$ & $\begin{array}{l}7 . \\
53\end{array}$ & $\begin{array}{c}7 . \\
52\end{array}$ & $\begin{array}{c}312 \\
.7\end{array}$ & $\begin{array}{c}321 \\
.5\end{array}$ & $\begin{array}{c}14 \\
.4\end{array}$ & $\begin{array}{c}14 . \\
8\end{array}$ \\
\hline $\mathrm{T}_{4}$ & $\begin{array}{c}20 \\
1 . \\
9\end{array}$ & $\begin{array}{c}206 . \\
5\end{array}$ & $\begin{array}{c}8 . \\
03\end{array}$ & $\begin{array}{l}8 . \\
12\end{array}$ & $\begin{array}{c}323 \\
.7\end{array}$ & $\begin{array}{c}326 \\
.5\end{array}$ & $\begin{array}{l}15 \\
.4\end{array}$ & $\begin{array}{c}15 . \\
9\end{array}$ \\
\hline $\mathrm{T}_{5}$ & $\begin{array}{l}16 \\
7 . \\
4\end{array}$ & $\begin{array}{c}168 . \\
9\end{array}$ & $\begin{array}{l}5 . \\
88\end{array}$ & $\begin{array}{c}6 . \\
12\end{array}$ & $\begin{array}{c}246 \\
.3\end{array}$ & $\begin{array}{c}252 \\
.3\end{array}$ & $\begin{array}{c}10 \\
.3\end{array}$ & $\begin{array}{c}10 . \\
8\end{array}$ \\
\hline $\mathrm{T}_{6}$ & $\begin{array}{c}19 \\
9 . \\
1\end{array}$ & $\begin{array}{c}205 . \\
6\end{array}$ & $\begin{array}{l}7 . \\
62\end{array}$ & $\begin{array}{l}7 . \\
62\end{array}$ & $\begin{array}{c}316 \\
.2\end{array}$ & $\begin{array}{c}320 \\
.1\end{array}$ & $\begin{array}{c}14 \\
.7\end{array}$ & $\begin{array}{c}15 . \\
0\end{array}$ \\
\hline $\mathrm{T}_{7}$ & $\begin{array}{l}20 \\
3 . \\
8\end{array}$ & $\begin{array}{c}210 . \\
3\end{array}$ & $\begin{array}{c}8 . \\
06\end{array}$ & $\begin{array}{l}8 . \\
21\end{array}$ & $\begin{array}{c}325 \\
.7\end{array}$ & $\begin{array}{c}328 \\
.9\end{array}$ & $\begin{array}{c}15 \\
.5\end{array}$ & $\begin{array}{c}16 . \\
0\end{array}$ \\
\hline $\mathrm{T}_{8}$ & $\begin{array}{l}18 \\
2 . \\
0\end{array}$ & $\begin{array}{c}185 . \\
4\end{array}$ & $\begin{array}{c}6 . \\
71\end{array}$ & $\begin{array}{c}6 . \\
82\end{array}$ & $\begin{array}{c}288 \\
.6\end{array}$ & $\begin{array}{c}292 \\
.3\end{array}$ & $\begin{array}{c}12 \\
.1\end{array}$ & $\begin{array}{c}12 . \\
8\end{array}$ \\
\hline $\mathrm{T}_{9}$ & $\begin{array}{l}17 \\
8 . \\
1\end{array}$ & $\begin{array}{c}182 . \\
6\end{array}$ & $\begin{array}{l}3 . \\
21\end{array}$ & $\begin{array}{c}6 . \\
32\end{array}$ & $\begin{array}{c}283 \\
.7\end{array}$ & $\begin{array}{c}285 \\
.6\end{array}$ & $\begin{array}{c}12 \\
.1\end{array}$ & $\begin{array}{c}12 \\
4\end{array}$ \\
\hline $\begin{array}{l}\text { S.E } \\
\text { m. } \pm\end{array}$ & $\begin{array}{l}5 . \\
9\end{array}$ & 5.2 & $\begin{array}{c}0 . \\
31\end{array}$ & $\begin{array}{l}0 . \\
41\end{array}$ & 9.0 & $\begin{array}{c}10 . \\
2\end{array}$ & $\begin{array}{l}0 . \\
42\end{array}$ & 0.4 \\
\hline $\begin{array}{l}\text { C.D. } \\
(P=0 \\
.05)\end{array}$ & $\begin{array}{l}17 \\
.8\end{array}$ & 15.5 & $\begin{array}{c}0 . \\
93\end{array}$ & $\begin{array}{c}1 . \\
21\end{array}$ & $\begin{array}{c}27 . \\
0\end{array}$ & $\begin{array}{c}30 . \\
4\end{array}$ & $\begin{array}{c}1 . \\
25\end{array}$ & 1.2 \\
\hline
\end{tabular}

The yield parameters viz., cob length, number of rows per cob, number of kernels per cob and 100 kernel weight were influenced significantly due to $\mathrm{N}$ management practices in both the experimental years (Table 2). Among the $\mathrm{N}$ management practices, SPAD sufficiency index 95-100 per cent recorded significantly higher cob length (15.5 and $16.0 \mathrm{~cm}$, respectively), number of rows per cob (16.7 and 17, respectively), number of kernels per cob (522 and 531, respectively) and 100 kernel weight (42.93 and 43.33, respectively) as compared to others. However, it was on par with $\mathrm{N}$ management through LCC 6, SPAD sufficiency index 90-95 per cent and LCC 5. This increased yield attributes was mainly attributed to increased growth parameters due to enhanced photosynthesis. These increased growth and yield attributes which in turn enhanced due to precise application of nitrogen based on the crop 
requirement similar results were found by $[12,14]$ in rice. While, lower yield attributes were recorded in LCC 3.

Table 2. Yield parameters of drip irrigated maize as influenced by precision nitrogen management practices

\begin{tabular}{|c|c|c|c|c|c|c|c|c|c|c|}
\hline $\begin{array}{c}\mathbf{T} \\
\mathbf{r} \\
\mathbf{e} \\
\mathbf{a t} \\
\mathbf{m}\end{array}$ & \multicolumn{2}{|c|}{$\begin{array}{c}\text { No. } \\
\text { of } \\
\text { rows } \\
\text { per } \\
\text { cob }\end{array}$} & \multicolumn{2}{|c|}{$\begin{array}{c}\text { No. } \\
\text { of ker } \\
\text { nels } \\
\text { per } \\
\text { cob }\end{array}$} & \multicolumn{2}{|c|}{$\begin{array}{c}100 \\
\text { ker } \\
\text { nel } \\
\text { wei } \\
\text { ght } \\
\text { (g) }\end{array}$} & \multicolumn{2}{|c|}{$\begin{array}{c}\text { Kernel } \\
\text { yie } \\
\text { ld } \\
\left(\mathrm{q} \mathrm{ha} \mathbf{~ h a}^{-1}\right)\end{array}$} & \multicolumn{2}{|c|}{$\begin{array}{c}\text { Stover } \\
\text { yi } \\
\text { eld } \\
\left(\mathbf{q} \mathbf{h a}^{-1}\right)\end{array}$} \\
\hline $\begin{array}{l}\mathbf{e} \\
\mathbf{n} \\
\mathbf{t} \\
\mathbf{s}\end{array}$ & $\begin{array}{l}2 \\
0 \\
1 \\
4\end{array}$ & $\begin{array}{l}\mathbf{2} \\
\mathbf{0} \\
\mathbf{1} \\
\mathbf{5}\end{array}$ & $\begin{array}{l}2 \\
0 \\
1 \\
4\end{array}$ & 1 & $\begin{array}{l}2 \\
0 \\
1 \\
4\end{array}$ & $\begin{array}{l}\mathbf{2} \\
\mathbf{0} \\
\mathbf{1} \\
\mathbf{5}\end{array}$ & $\begin{array}{l}2 \\
0 \\
1 \\
4\end{array}$ & $\begin{array}{l}\mathbf{2} \\
\mathbf{0} \\
\mathbf{1} \\
\mathbf{5}\end{array}$ & $\begin{array}{l}2 \\
0 \\
1 \\
4\end{array}$ & $\begin{array}{l}\mathbf{2} \\
\mathbf{0} \\
\mathbf{1} \\
\mathbf{5}\end{array}$ \\
\hline $\mathrm{T}_{1}$ & $\begin{array}{l}1 \\
1 . \\
0\end{array}$ & $\begin{array}{l}1 \\
1 . \\
5\end{array}$ & $\begin{array}{l}1 \\
8 \\
0\end{array}$ & $\begin{array}{l}1 \\
8 \\
5\end{array}$ & $\begin{array}{l}4 \\
1 . \\
1 \\
3\end{array}$ & $\begin{array}{l}4 \\
1 . \\
2 \\
3\end{array}$ & $\begin{array}{l}62 . \\
18\end{array}$ & $\begin{array}{l}6 \\
3 \\
. \\
4 \\
5\end{array}$ & $\begin{array}{l}9 \\
9 . \\
27\end{array}$ & $\begin{array}{l}1 \\
0 \\
0 \\
.8\end{array}$ \\
\hline $\mathrm{T}_{2}$ & $\begin{array}{l}1 \\
2 . \\
8\end{array}$ & $\begin{array}{l}1 \\
3 . \\
0\end{array}$ & $\begin{array}{l}2 \\
9 \\
1\end{array}$ & $\begin{array}{l}3 \\
0 \\
0\end{array}$ & $\begin{array}{l}4 \\
1 . \\
7 \\
3\end{array}$ & $\begin{array}{l}4 \\
1 . \\
8 \\
9\end{array}$ & $\begin{array}{l}70 . \\
54\end{array}$ & $\begin{array}{l}7 \\
2 \\
5 \\
5 \\
6\end{array}$ & $\begin{array}{l}1 \\
1 \\
0.4\end{array}$ & $\begin{array}{l}1 \\
1 \\
2 \\
.3\end{array}$ \\
\hline $\mathrm{T}_{3}$ & $\begin{array}{l}1 \\
6 . \\
1\end{array}$ & $\begin{array}{l}1 \\
6 . \\
5\end{array}$ & $\begin{array}{l}4 \\
9 \\
2\end{array}$ & $\begin{array}{l}4 \\
9 \\
5\end{array}$ & $\begin{array}{l}4 \\
2 . \\
6 \\
7\end{array}$ & $\begin{array}{l}4 \\
2 . \\
1\end{array}$ & $\begin{array}{l}77 . \\
92\end{array}$ & $\begin{array}{l}7 \\
8 \\
6 \\
6 \\
7\end{array}$ & $\begin{array}{l}1 \\
2 \\
1 . \\
9 \\
3\end{array}$ & $\begin{array}{l}1 \\
2 \\
5 \\
.7\end{array}$ \\
\hline $\mathrm{T}_{4}$ & $\begin{array}{l}1 \\
6 . \\
5\end{array}$ & $\begin{array}{l}1 \\
6 . \\
8\end{array}$ & $\begin{array}{l}5 \\
1 \\
7\end{array}$ & $\begin{array}{l}5 \\
2 \\
5\end{array}$ & $\begin{array}{l}4 \\
2 . \\
8 \\
7\end{array}$ & $\begin{array}{l}4 \\
2 . \\
6 \\
7\end{array}$ & $\begin{array}{l}85 . \\
27\end{array}$ & $\begin{array}{l}8 \\
6 \\
5 \\
7 \\
8\end{array}$ & $\begin{array}{l}13 \\
9 . \\
32\end{array}$ & $\begin{array}{l}1 \\
4 \\
2 \\
.7\end{array}$ \\
\hline $\mathrm{T}_{5}$ & $\begin{array}{l}1 \\
1 . \\
4\end{array}$ & $\begin{array}{l}1 \\
1 . \\
8\end{array}$ & $\begin{array}{l}2 \\
0 \\
7\end{array}$ & $\begin{array}{l}2 \\
1 \\
1\end{array}$ & $\begin{array}{l}4 \\
1 . \\
3 \\
3\end{array}$ & $\begin{array}{l}4 \\
1 . \\
8 \\
9\end{array}$ & $\begin{array}{l}63 . \\
39\end{array}$ & $\begin{array}{l}6 \\
5 \\
. \\
4 \\
3\end{array}$ & $\begin{array}{l}10 \\
0 . \\
97\end{array}$ & $\begin{array}{l}1 \\
0 \\
3 \\
.4\end{array}$ \\
\hline $\mathrm{T}_{6}$ & $\begin{array}{l}1 \\
6 . \\
2\end{array}$ & $\begin{array}{l}1 \\
6 . \\
6\end{array}$ & 4 & $\begin{array}{l}5 \\
0 \\
1\end{array}$ & $\begin{array}{l}4 \\
2 . \\
7 \\
3\end{array}$ & $\begin{array}{l}4 \\
3 . \\
2 \\
1\end{array}$ & $\begin{array}{l}78 . \\
23\end{array}$ & $\begin{array}{l}8 \\
0\end{array}$ & $\begin{array}{l}12 \\
7 . \\
52\end{array}$ & $\begin{array}{l}1 \\
3 \\
0 \\
.8\end{array}$ \\
\hline $\mathrm{T}_{7}$ & $\begin{array}{l}1 \\
6 . \\
7\end{array}$ & $\begin{array}{l}1 \\
7 . \\
0\end{array}$ & $\begin{array}{l}5 \\
2 \\
2\end{array}$ & $\begin{array}{l}5 \\
3 \\
1\end{array}$ & $\begin{array}{l}4 \\
2 . \\
9 \\
3\end{array}$ & $\begin{array}{l}4 \\
3 . \\
3 \\
3\end{array}$ & $\begin{array}{l}85 . \\
73\end{array}$ & $\begin{array}{l}8 \\
6 \\
. \\
7 \\
8\end{array}$ & $\begin{array}{l}14 \\
0.4\end{array}$ & $\begin{array}{l}1 \\
4 \\
5 \\
.3\end{array}$ \\
\hline $\mathrm{T}_{8}$ & $\begin{array}{l}1 \\
4 . \\
5\end{array}$ & $\begin{array}{l}1 \\
5 . \\
2\end{array}$ & $\begin{array}{l}4 \\
4 \\
6\end{array}$ & $\begin{array}{l}4 \\
6 \\
8\end{array}$ & $\begin{array}{l}4 \\
1 . \\
8 \\
3\end{array}$ & $\begin{array}{l}4 \\
2 . \\
1 \\
4\end{array}$ & $\begin{array}{l}72 . \\
5\end{array}$ & $\begin{array}{l}7 \\
3 \\
5 \\
4 \\
5\end{array}$ & $\begin{array}{l}11 \\
6 . \\
16\end{array}$ & $\begin{array}{l}1 \\
2 \\
0 \\
.2\end{array}$ \\
\hline $\mathrm{T}_{9}$ & $\begin{array}{l}1 \\
4 . \\
5\end{array}$ & $\begin{array}{l}1 \\
4 . \\
9\end{array}$ & $\begin{array}{l}4 \\
3 \\
3\end{array}$ & $\begin{array}{l}4 \\
4 \\
2\end{array}$ & $\begin{array}{l}4 \\
1 . \\
7\end{array}$ & $\begin{array}{l}4 \\
2 . \\
3\end{array}$ & $\begin{array}{l}70 . \\
83\end{array}$ & $\begin{array}{l}7 \\
2 \\
4 \\
4 \\
3\end{array}$ & $\begin{array}{l}11 \\
0.8 \\
1\end{array}$ & $\begin{array}{l}1 \\
1 \\
3 \\
.4\end{array}$ \\
\hline S. & 0. & 0 . & 1 & 1 & 0. & 0. & 2. & 2 & 3. & 3. \\
\hline
\end{tabular}

\begin{tabular}{|l|l|l|l|l|l|l|l|l|l|l|}
\hline Em. & 5 & 5 & 5. & 6. & 4 & 4 & 74 &. & 62 & 8 \\
\pm & 4 & 5 & 7 & 8 & 1 & 5 & & 8 & & 7 \\
& & & 8 & & & & & 2 & & \\
\hline C. & & & & & & & & & & \\
D. & 1. & 1. & 4 & 5 & & & & 8 & 1 & 1 \\
$(\mathrm{P}$ & 6 & 6 & 7. & 0. & $\mathrm{~N}$ & $\mathrm{~N}$ & 8. & 8 & 0. & 0. \\
$=$ & 3 & 6 & 3 & 9 & $\mathrm{~S}$ & $\mathrm{~S}$ & 2 & 5 & 8 & 5 \\
0. & & & 0 & & & & & & 4 & 8 \\
$05)$ & & & & & & & & & & \\
\hline
\end{tabular}

The higher kernel (85.73 and $86.78 \mathrm{q}^{-1}$ ha, respectively) and stover yield (140.40 and $145.3 \mathrm{q}^{-1}$ ha, respectively) was recorded under nitrogen management through SPAD sufficiency index 95-100 per cent as compared to other nitrogen management practices. However, it was at par with LCC 6 (85.27 and $86.78 \mathrm{q}$ $\mathrm{ha}^{-1}$, respectively), SPAD sufficiency index 90-95 per cent (78.23 and $80.12 \mathrm{q} \mathrm{ha}^{-1}$, respectively) and LCC 5 (77.92 and $78.67 \mathrm{q} \mathrm{ha}^{-1,}$ respectively). The extent of increase in the yield in the above treatments was 17.5, 17.0, 9.5 and 9.0 per cent, respectively over UAS (B) package (Table 2). The increase in the yield in these treatments was attributed due to application of right quantity of $\mathrm{N}$ fertilizer as per the crop demand and resulted in reduced losses lead to higher $\mathrm{N}$ use efficiency. The results are in agreement with the findings of [3] in maize; [5] in rice and [4] in wheat. The yield ability of the crop is the reflection of growth and yield attributing characters. The increase in kernel yield of maize could be traced back to increase in growth and yield attributes.

Economics is the ultimate criteria for acceptance and wider adoption of any technology. Among different indicators of economics efficiency in any production system, net returns and $\mathrm{B}: \mathrm{C}$ have greater impact on the practical utility and acceptance of the technology by the farmers. In the present study, comparative economics of precision nitrogen management practices are indicated. The economics of maize varied with respect to gross returns, which was a result of prices and yield of marketable produce, cost of cultivation which varies in relation to different inputs used, and in turn net returns and B:C. Among the various nitrogen management treatments nitrogen management through SPAD sufficiency index 95-100 per cent recorded higher gross returns (Rs.1,08, 264 and Rs. 1,16,444 $\mathrm{ha}^{-1}$, respectively), net returns (Rs. 69,634 and Rs. 77,614, respectively) and $\mathrm{B}: \mathrm{C}$ ratio (2.80 and 3.00 , respectively) (Table 3). Same trend was followed by former treatments with respect to net returns and benefit cost ratio in comparison with other precision nitrogen management practices. The consequence of higher yield and lower cost on $\mathrm{N}$ fertilizer resulted in higher $\mathrm{B}: \mathrm{C}$. This increased net returns and B:C in SPAD sufficiency index 95-100 per cent and LCC 6 was mainly due to increase in yield as well as reduction in the application of $\mathrm{N}$ fertilizer. These results are in agreement with the findings of [9, $11,4]$ in wheat.

Table 3. Economics of drip irrigated maize as influenced by precision nitrogen management practices 


\begin{tabular}{|c|c|c|c|c|c|c|c|c|}
\hline \multirow{2}{*}{$\begin{array}{l}\text { Tre } \\
\text { at } \\
\text { me } \\
\text { nts }\end{array}$} & \multicolumn{2}{|c|}{$\begin{array}{c}\text { Cost of } \\
\text { cultivati } \\
\text { on (Rs. } \\
\left.\text { ha }^{-1}\right)\end{array}$} & \multicolumn{2}{|c|}{$\begin{array}{c}\text { Gross } \\
\text { returns } \\
\text { (Rs. ha } \\
{ }^{1} \text { ) }\end{array}$} & \multicolumn{2}{|c|}{$\begin{array}{c}\text { Net } \\
\text { returns } \\
\left(\text { Rs. ha }{ }^{-1}\right)\end{array}$} & \multicolumn{2}{|c|}{ B:C } \\
\hline & $\begin{array}{l}20 \\
14\end{array}$ & $\begin{array}{l}20 \\
15\end{array}$ & $\begin{array}{l}20 \\
14\end{array}$ & $\begin{array}{l}20 \\
15\end{array}$ & 20 & 20 & $\begin{array}{l}20 \\
14\end{array}$ & $\begin{array}{l}20 \\
15\end{array}$ \\
\hline $\mathrm{T}_{1}$ & $\begin{array}{c}38 \\
01 \\
5\end{array}$ & $\begin{array}{c}38 \\
21 \\
5\end{array}$ & $\begin{array}{c}78 \\
38 \\
8\end{array}$ & $\begin{array}{c}84 \\
80 \\
0\end{array}$ & $\begin{array}{c}403 \\
73\end{array}$ & $\begin{array}{c}465 \\
85\end{array}$ & $\begin{array}{c}2.0 \\
6\end{array}$ & \\
\hline $\mathrm{T}_{2}$ & $\begin{array}{c}38 \\
18 \\
7\end{array}$ & $\begin{array}{c}38 \\
38 \\
7\end{array}$ & $\begin{array}{c}88 \\
82 \\
1\end{array}$ & $\begin{array}{c}96 \\
59 \\
3\end{array}$ & $\begin{array}{c}506 \\
34\end{array}$ & $\begin{array}{c}582 \\
06\end{array}$ & $\begin{array}{c}2.3 \\
3\end{array}$ & \\
\hline $\mathrm{T}_{3}$ & $\begin{array}{c}38 \\
25 \\
8\end{array}$ & $\begin{array}{c}45 \\
8\end{array}$ & $\begin{array}{c}98 \\
10 \\
8\end{array}$ & $\begin{array}{l}10 \\
56 \\
98\end{array}$ & $\begin{array}{c}598 \\
50\end{array}$ & $\begin{array}{c}672 \\
40\end{array}$ & $\begin{array}{c}2.5 \\
6\end{array}$ & \\
\hline $\mathrm{T}_{4}$ & $\begin{array}{c}38 \\
58 \\
0\end{array}$ & $\begin{array}{c}38 \\
78 \\
0\end{array}$ & $\begin{array}{l}10 \\
76 \\
53\end{array}$ & $\begin{array}{l}11 \\
61 \\
34\end{array}$ & $\begin{array}{c}690 \\
73\end{array}$ & $\begin{array}{c}773 \\
54\end{array}$ & $\begin{array}{c}2.7 \\
9\end{array}$ & \\
\hline $\mathrm{T}_{5}$ & $\begin{array}{c}38 \\
13 \\
7\end{array}$ & $\begin{array}{c}38 \\
33 \\
7\end{array}$ & $\begin{array}{c}79 \\
57 \\
6\end{array}$ & $\begin{array}{c}86 \\
95 \\
4\end{array}$ & $\begin{array}{c}414 \\
39\end{array}$ & $\begin{array}{c}486 \\
17\end{array}$ & $\begin{array}{c}2.0 \\
9\end{array}$ & $\begin{array}{l}2 . \\
27\end{array}$ \\
\hline $\mathrm{T}_{6}$ & $\begin{array}{c}38 \\
25 \\
8\end{array}$ & $\begin{array}{c}38 \\
45 \\
8\end{array}$ & $\begin{array}{c}98 \\
74 \\
7\end{array}$ & $\begin{array}{l}10 \\
68 \\
86\end{array}$ & $\begin{array}{c}604 \\
89\end{array}$ & $\begin{array}{c}684 \\
28\end{array}$ & $\begin{array}{c}2.5 \\
8\end{array}$ & $\begin{array}{l}2 . \\
78\end{array}$ \\
\hline $\mathrm{T}_{7}$ & $\begin{array}{c}38 \\
63 \\
0\end{array}$ & $\begin{array}{c}38 \\
83 \\
0\end{array}$ & $\begin{array}{l}10 \\
82 \\
64\end{array}$ & $\begin{array}{l}11 \\
64 \\
44\end{array}$ & $\begin{array}{c}696 \\
34\end{array}$ & $\begin{array}{c}776 \\
14\end{array}$ & $\begin{array}{c}2.8 \\
0\end{array}$ & \\
\hline $\mathrm{T}_{8}$ & $\begin{array}{c}36 \\
58 \\
1\end{array}$ & $\begin{array}{c}36 \\
78 \\
1\end{array}$ & $\begin{array}{c}91 \\
41 \\
9\end{array}$ & $\begin{array}{c}98 \\
51 \\
6\end{array}$ & $\begin{array}{c}548 \\
38\end{array}$ & $\begin{array}{c}617 \\
35\end{array}$ & $\begin{array}{c}2.5 \\
0\end{array}$ & $\begin{array}{l}2 . \\
68\end{array}$ \\
\hline $\mathrm{T}_{9}$ & $\begin{array}{c}36 \\
58 \\
1\end{array}$ & $\begin{array}{c}36 \\
78 \\
1\end{array}$ & $\begin{array}{c}89 \\
18 \\
3\end{array}$ & $\begin{array}{c}96 \\
70 \\
1\end{array}$ & $\begin{array}{c}526 \\
01\end{array}$ & $\begin{array}{c}526 \\
01\end{array}$ & $\begin{array}{c}2.4 \\
4\end{array}$ & $\begin{array}{l}2 . \\
63\end{array}$ \\
\hline
\end{tabular}

\section{CONCLUSIONS}

From the present study it is clear that nitrogen management through SPAD sufficiency index 90-100 per cent and LCC 5 and 6 helps in achieving higher yield and economics of maize under drip irrigated condition than UAS (B).

\section{References}

1. Anonymous, www.indiastat.com, Area production and productivity of major cereals (2018)

2. V. Balasubramanian, A.C. Morales, R.T. Cruz, T.M. Thiyagarajan, R. Nagarajan, M. Babu, S. Abdulrachman, L.H. Hai. Application of the chlorophyll meter (SPAD) technology for real-time $N$ management in rice Review, IRRI Notes, 25(1) 4$8(2000)$

3. M. Banerjee, Bhuiya, Gopal Sing, G.C. Malik, Dutta, Sudarshan. Precision nutrient management through use of LCC and nutrient expert in hybrid maize under laterite soil of India Universal Journal of Food and Nutritional Sciences, 2(2) 33-36 (2014)
4. M.S. El-Habbal, F. Ashmawy, H.S. Saoudi, K.H. Iman Abbas. Effect of nitrogen fertilizer rates on yield, yield components and grain quality measurements of wheat cultivars using SPAD meter Egypt Journal of Agricultural Research, 88(1) 14-18 (2010)

5. M. Ghosh, Swain, Dillip Kumar, Jha, Madan Kumar, Kumar, Virendra. Precision nitrogen management using chlorophyll meter for improving growth, productivity and $N$ use efficiency of rice in subtropical climate Journal of Agricultural Sciences, 5(2) 253-266 (2013).

6. K.A. Gomez, A.A. Gomez, Statistical procedures for agricultural research (2 ${ }^{\text {nd }}$ Edition Chichesler, UK:John Wiley and Sons 1984)

7. M.L. Jackson, Soil chemical analysis, Prentice Hall of India, Pvt Ltd New Delhi, p 498 (1973)

8. T. Jayanthi, S.K. Gali, V.P. Chimmad, V.V. Angadi, Leaf colour chart based $n$ management on yield, harvest index and partial factor productivity of rainfed rice Karnataka Journal of Agricultural Sciences, 20(2) 405-406. (2007)

9. K. Kenchaiah, H.K. Veeranna, K.M. Devaraju. India workshop cum group meeting in direct seeding and seeders in rice, Mysore, LCC and SPAD based-N management under different method of sowing in rice In abstract of the 3rd CREMNET,18-19 (2000)

10. N.R. Kitchen, K.A. Sudduth, S.T. Drummond, P.C. Scharf, H.L. Palm, D.F. Roberts, E.D. Vories. Ground based canopy reflectance sensing for variable-rate nitrogen corn fertilization Agronomy Journal, 10271 (2010)

11. D. Maiti, D.K. Das. Management of nitrogen through the use of leaf colour chart (LCC) and soil plant analysis development (SPAD) in wheat under irrigated ecosystem Archives Agronomy and Soil Science, 52(1) 105-112 (2006)

12. K. Manjappa, N. Kataraki, S.V. Kelaginamani. Leaf colour chart a simple tool for integrated nitrogen management in rainfed lowland rice. Karnataka Journal of Agricultural Sciences, 19(1) 84-89 (2006)

13. N.N. Singh, H. Pervez, Zaidi. Changes in priorities of maize research in India and relation to CIMMYT regional activities Maize Research India, pp 561570 (2002)

14. Singh, Jagdeep, C.S. Khind. Enhancing fertilizer nitrogen use efficiency in irrigated rice by using a chlorophyll meter and leaf colour chart The Bioscan, 9(1\&2) 663-666 (2015)

15. Thakur, Ashwani Kumar, Thakur, Dushyant Singh, Patel, Rakesh Kumar, Pradhan, Adikant and Kumar, Prafull. Effect of different plant geometry and nitrogen levels, in relation to growth characters, yield and economics on sweet corn (Zea mays Sachharata L.) at bastar plateau zone. The Bioscan, 10(3) 1223-1226 (2015) 